\title{
The Diagnostic Value of Pathology Examination in Clinically Unsuspected Lipomatous Soft-tissue Tumors
}

\author{
Marije Zwakman ${ }^{1}$, Judith Eleonora Katharina Regina Hentzen ${ }^{1}$, Agnes Marije Hoogland ${ }^{2}$, \\ José van der Starre-Gaal ${ }^{2}$, Engelbertus Gerardus Johannes Maria Pierik ${ }^{1}$, Anne Brecht Francken ${ }^{1}$ \\ ${ }^{1}$ Department of Surgery, ISALA, Zwolle, The Netherlands \\ ${ }^{2}$ Department of Pathology, ISALA, Zwolle, The Netherlands
}

Email address:

m.zwakman@isala.nl (M. Zwakman), j.e.k.r.hentzen@isala.nl (J. E. K. R. Hentzen), a.m.hoogland@isala.nl (A. M. Hoogland), j.gaal@isala.nl (J. van der Starre-Gaal), e.g.j.m.pierik@isala.nl (E. G. J. M. Pierik), a.b.francken@isala.nl (A. B. Francken)

\section{To cite this article:}

Marije Zwakman, Judith Eleonora Katharina Regina Hentzen, Agnes Marije Hoogland, José van der Starre-Gaal, Engelbertus Gerardus Johannes Maria Pierik, Anne Brecht Francken. The Diagnostic Value of Pathology Examination in Clinically Unsuspected Lipomatous Soft-tissue Tumors. Journal of Surgery. Vol. 9, No. 5, 2021, pp. 233-237. doi: 10.11648/j.js.20210905.14

Received: September 7, 2021; Accepted: September 22, 2021; Published: September 30, 2021

\begin{abstract}
Soft-tissue tumors range from benign to malignant tumors. Although these tumors are frequently unsuspected during surgical excision, additional pathology examination is usually performed in the absence of national guidelines in combination with the fear of missing a malignant tumor. The aim of this study is to investigate if it is safe to refrain from routinely performing pathology examinations of soft-tissue tumors when they are clinically unsuspected to have malignant origins. Data from all routinely performed pathology examinations from patients with one or more clinically unsuspected resected soft-tissue tumors between January 2018 and January 2021 were retrospectively extracted from a merged prospectively maintained institutional database. The primary outcome was the overall rate of premalignant and malignant soft-tissue tumors. Secondary outcomes included specific patient and tumor characteristics in patients with premalignant or malignant soft-tissue tumors and the healthcare costs associated with the performed pathology examinations. In this study, 1,035 resected soft-tissue tumors from 823 patients were analyzed. In total, 1,033 tumors (99.8\%) turned out to be benign during the pathology examination, and the remaining two tumors $(0.2 \%)$ were intermediate soft-tissue tumors without clinical consequences. Both intermediate soft-tissue tumors were larger than $10 \mathrm{~cm}$ during clinical examination and were larger than $5 \mathrm{~cm}$ during the final pathology examination. The estimated costs spent to perform these pathology examinations were $€ 44,009$. Refraining from pathology examination in unsuspected soft-tissue tumors smaller than $5 \mathrm{~cm}$ seems to be safe, as no malignant tumor was found in this large cohort of patients. In addition, a cost reduction of $€ 11,000$ per year can be achieved by refraining from pathology examinations in this specific group of small non-suspected soft-tissue tumors.
\end{abstract}

Keywords: Soft-tissue Tumors, Pathologic Examination, Healthcare Costs

\section{Introduction}

Lipomatous tumors are the most common type of soft-tissue tumors and in the majority of the cases present as slow-growing, nonpainful, well-defined, and mobile mass. Despite the fact that lipomatous soft-tissue tumors range from benign lipomas to aggressive, high-grade liposarcomas, the vast majority of these tumors turn out to be benign during final pathology examination.

Over the last three decades, premalignant and malignant soft-tissue tumors have been studied extensively, leading to increased knowledge and various worldwide recommendations regarding the preoperative evaluation and management of these rare tumors. The Dutch soft-tissue tumor guidelines recommend that any tumor larger than $5 \mathrm{~cm}$, lying deep in the fascia, or rapidly increasing in size should be regarded as malignant until proven otherwise [1]. Magnetic resonance imaging (MRI) can help differentiate malignant lipomatous tumors from benign ones, as many studies have reported direct correlations between imaging findings and pathological diagnosis [2-5].

However, in daily practice, physicians are mostly confronted with benign lipomatous soft-tissue tumors. 
Although these tumors are frequently unsuspected during surgical excision, additional pathology examination is routinely performed in the Netherlands in the absence of worldwide recommendations in combined with the fear of overlooking a malignant tumor.

The overall rate of premalignant and malignant findings during pathology examinations in patients with clinically unsuspected lipomas is still unknown. Nevertheless, performing a pathology examination may lead to a period of insecurity for the patient and an increase in healthcare costs. Therefore, the aim of this study is to investigate if it is safe to refrain from routinely performing pathology examinations of soft-tissue tumors when they are clinically unsuspected to have malign origins.

\section{Methods}

\subsection{Design, Setting, and Patients}

All consecutive patients that presented in the Isala Clinics, a large teaching hospital with 1,200 beds, with one or more clinically unsuspected soft-tissue tumors during local excisions from January 2018 to January 2021 were retrospectively identified from an institutional database from our pathology department. The study was approved by the Institutional Ethics Committee of the Isala Hospital (METC 210110).

In this current study, clinically suspected lipomas are defined as soft-tissue tumors that are clinically suspected to be lipomas before and during local excision (i.e., reported by the surgeon in the surgical procedure report, or suspected as lipoma in the pathology request). Patients with soft-tissue tumors with suspected malignancy during surgical excision are not presented in this manuscript since these specific tumors should always undergo a pathology examination.

\subsection{Preoperative Evaluation and Management}

All patients underwent a standardized preoperative screening to evaluate and stage these soft-tissue tumors. This screening consisted of reviewing the patients' oncologic history; clinical presentation at the outpatient clinic including the duration, growth and symptoms of the tumor; physical examination of the tumor including location, size, and mobility; local ultrasound in cases of unknown origin; and MRI for tumors larger than $5 \mathrm{~cm}$. Additional biopsies were only deemed necessary when the etiology of a soft-tissue tumor was not apparent based on its clinical and imaging features. Premalignant or malignant suspected soft-tissue tumors were discussed during the weekly meeting of an experienced, multidisciplinary team consisting of oncologic surgeons, medical oncologists, pathologists, radiologists, radiotherapists and, oncology nurses.

\subsection{Primary and Secondary Outcomes}

The primary outcome was the overall rate of premalignant and malignant soft-tissue tumors within this specific population. Secondary outcomes included the specific patient and tumor characteristics of the patients with premalignant or malignant soft-tissue tumors, the occurrence of postoperative complications, and the healthcare costs associated with the performed pathology examinations. Additional costs of telephone consultations with patients to discuss the pathology results are not included in the current study.

\subsection{Data Collection}

Data on patient characteristics, tumor characteristics, operative characteristics, postoperative morbidity, and pathology outcomes were collected retrospectively. Data on postoperative complications were collected up to 90 days after surgery and registered according to the Clavien-Dindo classification system [6]. All essential financial data for this study were collected retrospectively with assistance from the financial department. These data concerned the actual pathology costs that were declared to the health insurer. Data were collected and stored in compliance with the Declaration of Helsinki.

\subsection{Statistical Analyses}

All statistical analyses were conducted using SPSS $\AA$ Statistics version 26.0 (SPSS Inc., Chicago, IL, USA). Continuous variables with a normal distribution are expressed as mean (standard deviation [SD]) values, and those without a normal distribution are expressed as median (interquartile range [IQR]) values. Categorical variables are expressed as numbers and percentages. The cost-related analyses are carried out using 2019-2020 costs in euros.

\section{Results}

\subsection{Baseline Characteristics}

Eight hundred twenty-three patients underwent surgical excision of one or more soft-tissue tumors between January 2018 and January 2021. All 1,035 resected soft-tissue tumors were sent for additional pathology examination.

Table 1 provides an overview of patient and tumor characteristics for the entire cohort. One hundred eighty-one patients $(22.0 \%)$ had a history of previous lipomas and, 56 patients $(8 \%)$ had an oncological history. Most soft-tissue tumors were located in the trunk $(43.6 \%)$ and, were present for at least one year $(50.0 \%)$, and mechanical complaints $(21.5 \%)$, pain $(20.7 \%)$, and growth $(16.0 \%)$ were the most frequently reported symptoms. Preoperative diagnostics were performed 644 times; ultrasound (49.6\%) and MRI $(24.4 \%)$ were the most common used modalities. The median diameter during pathologic examination was $4.1 \mathrm{~cm}$ (IQR 2.5-6.5). Postoperative complications occurred in 18 patients $(2.0 \%)$, whereas in only two patients $(0.2 \%)$, a complication with a severe adverse event score of Clavien Dindo 3 occurred. In one case, a reoperation was indicated because of postoperative bleeding and the other case was complicated by seroma formation, which indicated a puncture under local anesthesia. 
Table 1. Patient and tumor characteristics, and perioperative outcomes of excised soft-tissue tumors in all 823 patients between January 2018 and January 2021.

\begin{tabular}{|c|c|}
\hline & Total \\
\hline \multicolumn{2}{|l|}{ Patient characteristics $(n=823)$} \\
\hline Age, $y \pm S D$ & $53.4(19.3)$ \\
\hline Female sex, n (\%) & $359(43.6)$ \\
\hline $\mathrm{BMI}, \mathrm{kg} / \mathrm{m}^{2} \pm \mathrm{SD}$ & $27.1(4.4)$ \\
\hline \multicolumn{2}{|l|}{ ASA, n $(\%)$} \\
\hline 1 & $468(56.9)$ \\
\hline 2 & $312(37.9)$ \\
\hline 3 & $29(3.5)$ \\
\hline \multicolumn{2}{|l|}{ Oncologic history, n (\%) } \\
\hline Breast cancer & $17(2.1)$ \\
\hline Skin cancer & $23(2.8)$ \\
\hline Sarcoma & $1(0.1)$ \\
\hline Other cancers & $25(3.0)$ \\
\hline History of resected lipoma, n (\%) & $181(22.0)$ \\
\hline \multicolumn{2}{|l|}{ Tumor characteristics $(n=1,035)$} \\
\hline \multicolumn{2}{|l|}{ Location of STT, n (\%) } \\
\hline Upper limb & $255(24.7)$ \\
\hline Lower limb & $104(10.1)$ \\
\hline Trunk & $450(43.6)$ \\
\hline Pelvic region & $49(4.8)$ \\
\hline Head & $73(7.1)$ \\
\hline Neck & $63(6.1)$ \\
\hline Throat & $37(3.6)$ \\
\hline \multicolumn{2}{|l|}{ Complaints, n (\%) } \\
\hline No complaints & $91(11.1)$ \\
\hline Mechanical complaints & $177(21.5)$ \\
\hline Pain & $170(20.7)$ \\
\hline Growth & $132(16.0)$ \\
\hline Cosmesis & $84(10.2)$ \\
\hline Fear of cancer & $29(3.5)$ \\
\hline Loss of sensibility & $9(1.1)$ \\
\hline Itching & $2(0.2)$ \\
\hline Unknown & $129(15.7)$ \\
\hline Estimated diameter STT $(\mathrm{cm})+$ SD & $4.5(2.9)$ \\
\hline Diameter STT pathologic report (cm, IQR) & $4.0(2.5-6.5)$ \\
\hline \multicolumn{2}{|l|}{ Preoperative diagnostics, $\mathrm{n}(\%)$} \\
\hline Ultrasound & $412(50.1)$ \\
\hline $\mathrm{CT}$ & $7(0.9)$ \\
\hline MRI & $199(24.2)$ \\
\hline Cytology & $12(1.5)$ \\
\hline Histology & $14(1.7)$ \\
\hline \multicolumn{2}{|l|}{ Perioperative outcomes $(\mathrm{n}=823)$} \\
\hline Excision under local anaesthesia, n (\%) & $520(63.2)$ \\
\hline Re-excision, $\mathrm{n}(\%)$ & $3(0.4)$ \\
\hline \multicolumn{2}{|l|}{ SAE score, $\mathrm{n}(\%)$} \\
\hline $1-2$ & $16(1.8)$ \\
\hline 3 & $2(0.2)$ \\
\hline \multicolumn{2}{|l|}{ Grade 3 complications, $\mathrm{n}(\%)$} \\
\hline Wound infection & $1(0.1)$ \\
\hline Postoperative bleeding & $1(0.1)$ \\
\hline
\end{tabular}

$A S A$ American Society of Anesthesiology, STT soft tissue tumor, $C T$ computed tomography, $M R I$ magnetic resonance imaging, $S A E$ severe adverse event.

\subsection{Benign Soft-tissue Outcomes}

One thousand thirty-three out of 1,035 resected soft-tissue tumors $(99.8 \%)$ were ultimately found to be benign during the final pathology examination (Table 2). The most frequently found benign soft-tissue tumors were lipomas $(71.8 \%)$ and angiolipomas $(23.6 \%)$.
Table 2. Pathologic outcomes for all 1,035 soft-tissue tumors, $n(\%)$.

\begin{tabular}{ll}
\hline Benign STT & \\
\hline Lipoma & $743(71.8)$ \\
Angiolipoma & $244(23.6)$ \\
Spindle cell lipoma & $29(2.8)$ \\
Nuchal lipoma & $7(0.7)$ \\
Fibrolipoma & $3(0.3)$ \\
Fat necrosis & $2(0.2)$ \\
Pleiomorphic lipoma & $2(0.2)$ \\
Pilar cyst & $2(0.2)$ \\
Leiomyoma & $1(0.1)$ \\
Naevus lipomatosus & $1(0.1)$ \\
Intermediate STT & \\
Atypical lipomatous tumor (ALT) & $2(0.2)$ \\
\hline
\end{tabular}

STT soft tissue tumor.

\subsection{Malignant Soft-tissue Outcomes}

None of the 1,035 resected soft-tissue tumors turned out to be malignant during the final pathology examination. Only two atypical lipomatous soft-tissue tumors were found within this population; this kind of tumor is defined as an intermediate soft-tissue tumor, according to the Dutch guideline [1]. This specific type of tumor has a locally aggressive character but rarely metastasizes to other organs, and therefore, locally radical resection is the appropriate treatment.

The first case describes a female of 71 years old, with a medical history of breast cancer, who presented with a soft-tissue tumor on her upper limb. It was present for less than three months. During physical examination, the tumor was measured up to $10 \mathrm{~cm}$. An ultrasound and additionally an MRI found an intramuscular tumor of $8.6 \mathrm{~cm}$ suspected to be a sarcoma. A fine needle biopsy was performed that only showed benign cells of an intramuscular lipoma. Surgical resection of the tumor was performed, and the surgeon reported an atypical lipomatous tumor of $16 \mathrm{~cm}$ in the surgical procedure report. In both lesions, the tumor was excised radical, no additional treatment was performed and no complications were reported.

The second case describes a male of 50 years old, with a medical history of multiple lipoma's, who presented with a soft-tissue tumor on his lower limb. During physical examination, the tumor was measured up to $10 \mathrm{~cm}$. An MRI showed an intramuscular tumor of $6.5 \mathrm{~cm}$ suspected to be a lipomatous tumor. Surgical resection of the tumor was performed, and during the pathology examination, an atypical lipomatous tumor of $7.5 \mathrm{~cm}$ was found. No additional treatment was performed, and no complications were reported.

\subsection{Healthcare Costs}

In total, during the final pathology examination, 627 soft-tissue tumors were smaller than $5 \mathrm{~cm}(60.6 \%), 312$ soft-tissue tumors were $5 \mathrm{~cm}$ or larger $(30.1 \%)$, and in 96 tumors $(9.3 \%)$, no size was mentioned in the pathology report. The costs for the pathology examination included $€ 41.65$ per case for soft-tissue tumors smaller than $5 \mathrm{~cm}$ and $€ 44.27$ per case for soft-tissue tumors of at least $5 \mathrm{~cm}$. Therefore, for the entire cohort, at least $€ 44,009$ was spent on pathologic 
examinations, taking into account that the 96 soft-tissue tumors of unknown size cost $€ 41.65$ per pathology examination.

All small soft-tissue tumors (i.e., smaller than $5 \mathrm{~cm}$ ) were benign during the final pathology examination. In retrospect, an estimated amount of $€ 26,115$ was spent on pathologic examinations for unsuspected soft-tissue tumors smaller than $5 \mathrm{~cm}$.

\section{Conclusion}

This retrospective observational study, including 823 patients with 1,035 clinically unsuspected resected soft-tissue tumors in total, showed that during final pathology examination only two intermediate soft-tissue tumors were found without clinical consequences. All remaining soft-tissue tumors were ultimately found to be benign. For this specific population, an estimated $€ 44,000$ in healthcare costs could be saved by refraining from these pathology examinations in patients with unsuspected soft-tissue tumors.

\section{Discussion}

In our current cohort, $99.8 \%$ of the resected soft-tissue tumors turned out to be benign during the final pathology examination, and the remaining $0.02 \%$ represented intermediate soft-tissue tumors that were radically resected during primary surgery; therefore, pathology results did not have any additional clinical consequences. From an oncologic perspective, this high percentage of benign tumors within our current cohort can be explained by the fact that these patients had low risk to have malignancy, as at least 412 patients $(50.1 \%)$ had complaints for more than one year, 181 patients $(22.0 \%)$ had had resected lipomas in the past, and only 66 patients $(8.0 \%)$ had an oncologic history, including 23 patients $(2.8 \%)$ with skin cancers. In the majority of the cases, radiology was performed preoperatively consisting of an ultrasound in $50.1 \%$ of the cases and an MRI in $24.2 \%$ of the cases. Of course, the most important explanation of our current results is the fact that this cohort consists of patients with resected soft-tissue tumors that were clinically unsuspected during resection. Nevertheless, the aim of the current study is to investigate if it is safe to refrain from pathology examination in patients with clinically unsuspected soft-tissue tumors. This study shows for the first time that it might be safe to refrain from these pathology examinations for patients with clinically unsuspected soft-tissue tumors smaller than $5 \mathrm{~cm}$.

To the best of our knowledge, no previous study has examined the incidence of malignancy in patients with unsuspected soft-tissue tumors. McClintock et al., in a retrospective study, examined the necessity of core needle biopsy in resected lipomatous masses on the extremities or trunk in one single center between 2014 and 2017. In 178 patients, the authors found only two malignant tumors. Both patients with malignant soft-tissue tumors had tumors larger than $5 \mathrm{~cm}$, completed pre-operative imaging, and underwent core needle biopsy prior to excision [7]. However, no data on the clinical judgment of the surgeon, and thus if these tumors were suspected during resection, were reported. Three retrospective studies examined characteristics to differentiate large lipomas from atypical lipomatous tumors. Multivariate analyses showed that the size of a soft-tissue tumor larger than $10 \mathrm{~cm}$ was significantly associated with the risk of being diagnosed with an intermediate soft-tissue tumor [8-10]. It should be noted that none of these studies focused on whether pathologic examination could be omitted. Thus, it remains challenging to compare the results of this present study with the mentioned data from the current scientific literature.

The main reason to perform pathologic examination is to confirm the origin of the resected tumor and the clinical consequences for additional therapy. Refraining from pathology examination might introduce uncertainty in patients and doctors. One should always consider that a malignancy with unknown origins could appear in the future and that the previously resected soft-tissue tumor could be the primary tumor. Therefore, shared decision-making with the patient in the outpatient clinic is of the utmost importance. Nevertheless, insecurity might also occur in patients when the soft-tissue tumor is not resected. One could question whether surgical resection, instead of follow-up, is necessary when a soft-tissue tumor appears to be benign clinically and sometimes radiologically. However, follow-up would increase healthcare costs because more clinical visits would be needed, and in almost all cases within our cohort, patients had complaints such as mechanical complaints, pain, fear of cancer, growth, or cosmesis, and therefore requested to remove the tumor.

As mentioned previously, within our cohort, radiologic examinations were frequently performed prior to excision contributing to a further increase in healthcare costs for benign lesions. MRI, especially is an expensive tool, with an estimated cost of $€ 200$ in our hospital. According to the guideline, alarm symptoms are an indication for further analyses through radiologic examination. A soft-tissue tumor larger than $5 \mathrm{~cm}$ is considered an alarm symptom [11]. In the diagnostic process, ultrasound has no role that would change treatment when there is suspicion of a malignancy [12]. However, many lesions are unsuspicious but of uncertain exact location when it comes to the relation to muscular fascia or intramuscular location. For this specific question, ultrasound can be a helpful as it is a simple, effective diagnostic tool with low costs. An MRI is the best modality to visualize the extent of the soft-tissue tumor and a useful tool for preoperative imaging and preparation for surgical resection because important blood vessels and nerves can be identified preoperatively [13-15]. MRI is performed when the soft-tissue tumor appears to be larger than $5 \mathrm{~cm}$. From a financial perspective, omitting ultrasound in soft-tissue tumors clinically unsuspected for malignancy, should reduce healthcare costs. It should be noted that ultrasound in the Netherlands is mostly requested by the general practitioner and is therefore already performed before the patient visits the hospital. Since all patients were included retrospectively, this study could not make reliable statements regarding the usefulness of the performed radiologic examination to distinguish benign tumors from malignant ones. A prospective 
study about the exact structure of costs, and the necessity of performed preoperative imaging, might further assist in creating a valued-based guideline on the use of radiologic examination on soft-tissue tumors.

This study has certain strengths and limitations. To the best of our knowledge, this is the first study that specifically describes the diagnostic value of pathologic examination in soft-tissue tumors. No cost-effectiveness analyses on soft-tissue tumors have been published before, which demonstrates the importance of this study. Our cohort consists of a large total of patients with, in total, 1,035 resected soft-tissue tumors. All soft-tissue tumors were clinically and systematically assessed according to the guideline prior to resection. On the other hand, our study has some limitations due to its retrospective design and single-center approach. The healthcare costs of only one center were available; therefore, it could be possible that this study cannot assume that other centers would have the same amount of saved costs, although health insurers in the Netherlands prevent much variation in costs between medical centers. Moreover, it should be taken into account that the total costs of the procedure include not only the pathologic examination but also the surgical procedure, costs extracted from complications, radiology costs, and costs from outpatient visits, which are not included in our current study.

In conclusion, this study shows for the first time that refraining from pathologic examination might be safe in clinically unsuspected lipomatous soft-tissue tumors smaller than $5 \mathrm{~cm}$ and therefore will contribute to reducing healthcare costs. Prospective studies about the exact structure of costs, the necessity of performed preoperative imaging, and the direct feedback of surgeons regarding the risk of malignancy might further assist in future healthcare policy decisions and in creating a valued-based guideline on sending soft-tissue tumors for pathologic examination.

\section{References}

[1] www.richtlijnendatabase.nl; Wekedelentumoren. Kennisinstituut van de Federatie van Medisch Specialisten. 01-11-2011.

[2] El Ouni F, Jemni H, Trabelsi A, Ben Maitig M, Arifa N, Ben Rhouma K, et al. Liposarcoma of the extremities: MR imaging features and their correlation with pathologic data. Orthop Traumatol Surg Res 2010; 96: 876-83.
[3] Toirkens J, De Schepper A, Vanhoenacker F, Van Dyck, et al. A comparison between histopathology and findings on magnetic resonance imaging of subcutaneous lipomatous soft-tissue tumors. Insights Imaging 2011; 2: 599-607.

[4] Brisson M, Kashima T, Delaney D, Tirabosco R, Clarke A, Cro $\mathrm{S}$, et al. MRI characteristics of lipoma and atypical lipomatous tumour/well-differentiated liposarcoma: retrospective comparison with histology and MDM2 gene amplification. Skeletal Radiol 2013; 42: 635-47.

[5] Nagano S, Yokouchi M, Setoguchi T, Ishidou Y, Sasaki H, Shimada $\mathrm{H}$, et al. Differentiation of lipoma and atypical lipomatous tumour by a scoring system: implication of increased vascularity on pathogenesis of liposarcoma. BMC Musculoskelet Disord 2015; 16: 36.

[6] Clavien PA, Barkun J, de Oliveira ML, Vauthey JN, Dindo D, Schulick RD et al. The Clavien-Dindo classification of surgical complications: five-year experience. Ann Surg 2009; 250: $187-196$.

[7] McClintock NC, Satyananda V, Dauphine C, Hari DM, Chen KT, Ozao-Choy JJ. Lipomatous Soft Tissue Masses: Challenging the Paradigm of Routine Preoperative Biopsy. Journal of Surgical Research 2020; 247: 103-107.

[8] Bird JE, Morse LJ, Feng L, Wang WL, Lin PP, Moon BS, Lazar AJ, Satcher RL, Madewell JE, Lewis VO. Non-Radiographic Risk Factors Differentiating Atypical Lipomatous Tumors from Lipomas. Oncol. 2016; 6: 197.

[9] Fisher SB, Baxter KJ, Staly CA, Fister KE, Monson DK, Murray DR, Oskouei SV, Weiss SW, Kooby DAA, Maithel SK, Delman KA. The General Surgeon's Quandary: Atypical Lipomatous Tumor vs Lipoma, Who Needs a Surgical Oncologist? J Am Coll Surg. 2013; 217 (5): 881-8.

[10] Myhre-Jensen O. A consecutive 7-year series of 1331 benign soft tissue tumours. Clinicopathologic data. Comparison with sarcomas. Acta Orthop Scand. 1981; 52 (3): 287-93.

[11] Cooper CS, Stratton MR. Soft tissue tumors: the genetic basis of development. Carcinogenesis. 1991; 12 (2): 155-65.

[12] Fornage BD. Soft tissues massas: the underutilization of sonography. Semin Musculoskelet Radiol. 1999; 3 (2): 115-33.

[13] Ma LD. MRI of muscoskeletal tumors: skeletal and soft tissue masses. Curr Probl Diagn Radiol. 1999; 28 (2): 29-62.

[14] Sundaram M. MRI of soft tissue tumors: an overview. Semin Muscoloskelet Radiol. 1999; 3 (1): 15-20.

[15] Petterson $\mathrm{H}$, et al. Primary musculoskeletal tumors: examination with MRI compared with conventional modalities. Radiology. 1987; 164 (1): 237-41. 